\title{
Análise estrutural da floresta tropical úmida do município de Alta Floresta, Mato Grosso, Brasil
}

\author{
Antonio Francisco MALHEIROS ${ }^{1}$, Niro HIGUCHI ${ }^{2}$, Joaquim dos SANTOS $^{3}$ \\ RESUMO \\ Este trabalho teve como objetivo estudar a composição e estrutura de um estrato arbóreo da floresta tropical úmida em Alta \\ Floresta - MT, determinando os padrões de semelhança com outras regiões da Amazônia brasileira. O estudo foi realizado em \\ uma área de 2 hectares, dividida em 20 parcelas de 10x 100m, onde foram mensurados a altura e o DAP $\geq 10 \mathrm{~cm}$ de todos \\ os indivíduos. Para avaliação do grau de similaridade entre a composição florística e estrutura arbórea de Alta Floresta com as \\ nove regiōes pertencentes à Amazônia Legal brasileira, utilizou-se o método de agrupamento hierárquico aglomerativo com \\ ligaçôes pela média dos grupos (UPGMA), por meio do índice de Sorensen (qualitativo). Para a ordenação, foi utilizado o \\ mesmo programa, utilizando a Escalonamento Multidimensional não-métrica (NMS) por meio do índice de Jaccard aplicado \\ à presença e ausência de famílias e gêneros. Para análise de similaridade entre as 10 regiōes, comparando a matriz de distâncias \\ físicas entre elas com as matrizes de composição de famílias e gêneros, utilizou-se o programa PATN por meio do Teste de \\ Mantel com o índice de Jaccard. A região apresentou 1101 indivíduos, pertencentes a 32 famílias, 54 gêneros e 68 espécies. \\ A família com maior riqueza de espécie foi Leguminosae. A espécie Helicostilys podogyne e o gênero Cecropia sp. foram as mais \\ importantes no levantamento. A floresta tropical úmida de Alta Floresta não se assemelhou com nenhuma das nove regióes \\ comparadas neste estudo.
}

PALAVRAS-CHAVE: Composição florística, Estrutura, Similaridade, Amazônia mato-grossense, Alta Floresta.

\section{Structural analysis of tropical rainforest in Alta Floresta city, Mato Grosso, Brazil}

\section{ABSTRACT}

This paper deals with the composition and structure of a pristine tropical rainforest using likelihood patterns to compare it with other Amazonian sites. This work was carried out in Alta Floresta, Mato Grosso. Our ground truth was obtained from a two-hectare plot, which was divided into 20 plots of 10 by $100 \mathrm{~m}$ each. In each plot, all trees with $\mathrm{dbh}=10 \mathrm{~cm}$ were recorded. To evaluate the level of similarity between the Alta Floresta and other nine Amazonian sites we used the hierarchy clustering method linked by group means (UPGMA) through Sorensen index (qualitative). For ordination, we used the same method based on multidimensional non-metric scaling (NMS) through Jaccard index applied to presence or absence of botanical families or genera. The software PATN was used to run Mantel test with Jaccard index, which was, in turn, used for the similarity analysis among sites. The studied site presented 1,101 individuals distributed in 32 botanical families, 54 genera e 68 species. The richest family was Leguminosae. Helicostilys podogyne and Cecropia sp. were the most important species of the studied area. The Alta Floresta site did not present any similarity when compared to other Amazonian sites.

KEY WORDS: Floristic composition, Structure, Similarity, Amazonian mato-grossense, Alta Floresta.

\footnotetext{
1 Universidade do Estado de Mato Grosso - UNEMAT. Av. São João, S/N - Cavalhada - Cáceres-MT. 78200-000. E-mail: afmalheiros@usp.br

2 Instituto Nacional de Pesquisas da Amazônia, Coordenação de Pesquisas em Silvicultura Tropical. E-mail: niro@inpa.gov.br

${ }^{3}$ Instituto Nacional de Pesquisas da Amazônia, Coordenação de Pesquisas em Silvicultura Tropical. E-mail: joca@inpa.gov.br
} 


\section{INTRODUÇÃO}

As florestas tropicais não apresentam um verdadeiro equilíbrio entre as comunidades vegetais, no qual dois indivíduos próximos dificilmente pertençam a uma mesma espécie (Hubbel \& Foster, 1986)

Por sua vez, tenderiam a ser mais generalistas, ocasionando desse modo, flutuações nas abundâncias das populaçôes (Hubbel, 1979), sendo isso, talvez, um dos fatores que explicam a alta diversidade, característica esta, da floresta Amazônica.

A caracterização da região amazônica é muito importante para definir a diversidade vegetacional, permitindo, dessa maneira, saber quais são as espécies que compõem tais áreas, e de que modo elas estão distribuídas num estrato arbóreo.

Conforme Rossi \& Higuchi (1998) os dados quantitativos da floresta podem ser descritos por meio de sua análise estrutural, juntamente com os padrōes de distribuição espacial, de crescimento e composição de algumas espécies.

Vieira \& Hosokawa (1989) comentam que a caracterização precisa de uma comunidade vegetal que permita sua identificação e distribuição, podendo dessa maneira comparála com outras associaçôes vegetais.

De acordo com Cesar \& Leitão-Filho (1990) a análise fitossociológica busca os estudos comparativos entre comunidades florestais visando comparaçōes em nível de espécies e sua importância nos diferentes compartimentos da floresta.

Os estudos com relação à similaridade entre as regiōes Amazônicas foram realizados por Gentry \& Emmons (1987), que compararam 13 regiōes em 6 países da Amazônia, utilizando variáveis ambientais, como a taxa de precipitação e tipos de solos de cada local, demonstrando a correlação existente entre as diferentes composiçōes da floresta de subbosque. Terborgh \& Andresen (1998) obtiveram um padrão em escala regional da composição florística, analisando a similaridade entre 29 locais em 7 países da Amazônia, entre a distância física e a média do número de indivíduos de cada família.

Oliveira (1997) comparou a existência de relaçôes florísticas e padrões de estrutura de uma floresta de vegetação de terra firme da Amazônia, localizada em Manaus, com16 trabalhos disponíveis em literatura, e observou que a similaridade florística entre as parcelas próximas é maior do que entre parcelas mais distantes, destacando a existência de uma alta afinidade com as áreas a leste e oeste da Amazônia, observando que a vegetação estudada de Manaus é uma área de confluência de províncias fitogeográficas da Amazônia.

Um levantamento da flora de um estrato arbóreo, em uma zona de tensão ecológica entre a floresta Amazônica e o cerrado, é muito importante para que possamos verificar a similaridade com outras regiōes da Amazônia, em relação à composição e estrutura, por meio de comparação com diversos trabalhos realizados na Amazônia Brasileira, disponíveis na literatura, podendo dessa forma observar qual é o padrão vegetacional que mais a região se assemelha e estabelecer expectativa de exploração.

Neste sentido, este trabalho teve como objetivo estudar a composição e estrutura de um estrato arbóreo da floresta tropical úmida em Alta Floresta -MT, determinando os padrões de semelhança com outras regiōes da Amazônia brasileira.

\section{MATERIAL E MÉTODOS}

\section{ÁREA DE ESTUDO}

O estudo foi desenvolvido na Fazenda Caiabi, localizada à latitude de $09^{\circ} 56^{\prime} 40^{\prime \prime} \mathrm{S}$ e longitude de $56^{\circ} 20^{\prime} 10^{\prime \prime} \mathrm{W}$, ocupando uma área de 12.000 ha, situada à, aproximadamente, $28 \mathrm{~km}$ a Oeste do município de Alta Floresta-MT (Figura 1), e este à $800 \mathrm{~km}$ ao norte de Cuiabá-MT, ligados pelas rodovias BR 163 e MT 208. A fazenda está limitada pelo rio Santa Helena a leste, e pelo córrego do Neguinho ao sul.

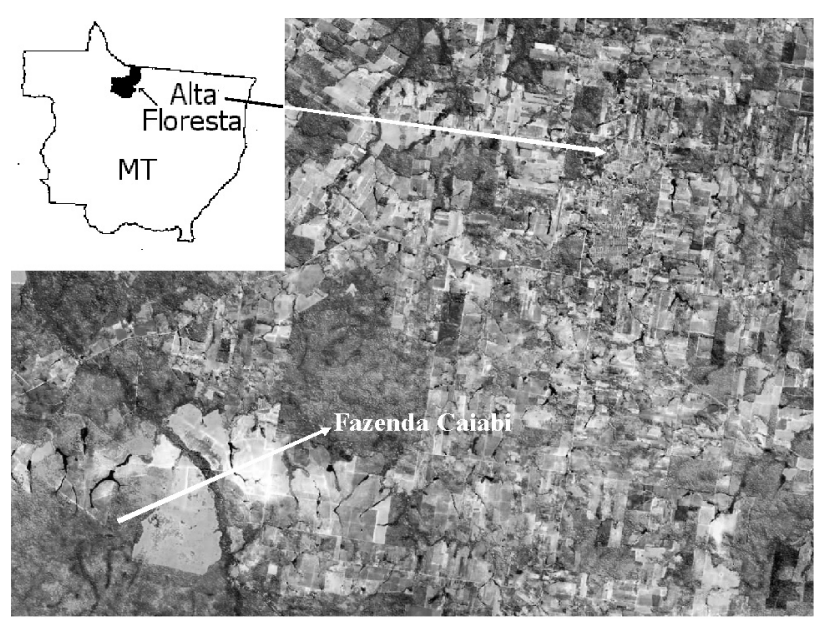

Figura 1 - Localização do município de Alta Floresta e da fazenda Caiabi (local do experimento), estado do Mato Grosso, Brasil. Fonte: Malheiros, 2000.

A região de Alta Floresta apresenta clima tropical quente úmido, com temperaturas médias elevadas $\left(23\right.$ e $\left.26^{\circ} \mathrm{C}\right)$ durante o ano, com máximas diárias de 34 a $37^{\circ} \mathrm{C}$. Não raramente, registram-se temperaturas próximas de $40^{\circ} \mathrm{C}$ (IBGE, 1988).

Os tipos de solos predominantes em Alta Floresta-MT são os Argilossolos Vermelho-Amarelo Distrófico, ocorrendo, como subdominante na maioria das manchas, latossolo vermelho-amarelo e latossolo amarelo (IBGE, 1999). Os compartimentos do relevo da região correspondem ao Planalto Meridional do Sul a Amazônia. Os domínios morfoestruturais 
compreendem as Bacias e Coberturas Sedimentares Fanerozóicas. a (IBGE, 2006).

Os tipos vegetacionais que ocorrem no município são: floresta ombrófila aberta tropical, floresta densa tropical, savanas e áreas de tensão ecológica (Loureiro et. al., 1980). Sánchez (1992) descreveu duas eco-regiōes para a vegetação do norte do Estado de Mato Grosso: Florestas pluviais das baixadas e serras e planaltos residuais do norte de Mato Grosso (Planalto dos Pareci). A Fazenda Caiabi está coberta por floresta ombrófila aberta tropical.

\section{COLETA DE DADOS}

Para coleta de dados foram utilizadas duas parcelas com dimensões de 100mx 100m, denominadas de área A e B, com uma distância de $100 \mathrm{~m}$ entre elas, situadas em platô. Este desenho experimental faz parte do Projeto multidisciplinar: "Combustão de Material de Diferentes Tamanhos em Queimadas de Florestas Tropicais" - FAPESP/INPE/UNESP/ Forest Service Department of Agriculture/Universidade de Washington/UNB.

Todos os indivíduos com DAP $\geq 10 \mathrm{~cm}$ tiveram anotado as medidas de circunferência à altura do peito. Para a identificação das plantas, foi coletado o material estéril e/ou fértil, em seguida foram anotados os nomes vulgares fornecidos por um assistente de campo. Para identificação botânica do material coletado foi utilizada literatura especializada (Parrotta et al. 1995; Mabberley, 1981; Ribeiro et al. 1999; INPA/CPPF, 1991; Rizzini, 1971; Pott \& Pott, 1994; Lorenzi, 1992; Joly, 1991), e por profissionais dos herbários da UFMT e do INPA. As exsicatas foram depositadas no herbário da UFMT.

A análise fitossociológica foi feita de acordo com a metodologia usada por Jardim \& Hosokawa (1986) e Ribeiro et al. (1993). Esta metodologia utiliza parâmetros das espécies como: Frequência, Densidade e Dominância, colocados de modo relativo e agrupados em um único valor que indica a importância ecológica (IVI) da espécie na floresta.

Santos (1996) destacou que a ordem de importância de espécies pode ser expressa pelo índice valor de cobertura (IVC), que expressa a somatória da densidade relativa e dominância relativa. Esses dados foram analisados utilizando o programa FITOPAC 1 (Shepherd, 1994).

Para verificar a similaridade foram utilizados nove estudos da Amazônia Legal Brasileira, disponíveis em literatura, que apresentam densidade absoluta e abundância das espécies que ocorreram em cada local e DAP com valor igual a $10 \mathrm{~cm}$ de diâmetro.

Pelo fato de haver diferenças no tamanho das amostras em alguns desses trabalhos foi empregada a média do número de indivíduos/ha.
Uma matriz de presença/ausência de gêneros e outra de famílias foram construídas, bem como de densidade absoluta dos gêneros e das famílias para os dados da Fazenda Caiabi, como também, para os dados das nove áreas da Amazônia, baseados na literatura, com a finalidade de comparar as relaçôes florísticas e padrões de estrutura vegetacionais entre as regióes analisadas.

A montagem das matrizes foi realizada no programa SYSTAT (Wilkinson, 1990). Essas matrizes foram comparadas com uma outra matriz de distância entre as 10 regiōes (incluindo este trabalho), analisadas neste trabalho por meio do PATN (Belbin, 1995), utilizando o Mantel Test (Fortin \& Gurevitch, 1993). Isto possibilitou examinar a correlação existente entre duas matrizes, onde uma representa a distância física entre as amostras e outra representa as diferenças na composição florística das regiôes estudadas.

Para responder à questão de similaridade utilizou-se o método de agrupamento hierárquico aglomerativo com ligaçōes pela média dos grupos (UPGMA), o qual consiste em calcular a média aritmética da similaridade (ou distância) entre o objeto que se quer incluir num grupo e cada objeto desse grupo, usando os índices qualitativos de Sorensen, considerando a presença e ausência dos gêneros e famílias de cada local (Ruokolainen \& Tuornisto, 1998). A ordenação foi feita por meio de análises de MNS (Escalonamento Multidirnensional não-métrico) e do índice de Jaccard aplicados à presença e ausência e gêneros e famílias, permitindo analisar as relaçôes entre amostras e gêneros a partir de uma única análise (Valentin, 1995). Para aplicação dos índices foi utilizado o programa PC-ORD (McCune \& Mefford, 1995).

\section{RESULTADOS}

Na Fazenda Caiabi em Alta Floresta-MT, as duas parcelas de 1 ha cada denominadas de área A e B, apresentaram 594 e 507 indivíduos respectivamente. A distribuição diamétrica dos 1101 indivíduos em relação às classes de $\mathrm{DAP} \geq 10 \mathrm{~cm}$, apresenta a forma de um J "invertido" (Figura 2).

Apenas seis indivíduos pertencentes a três espécies apresentaram DAP $>100 \mathrm{~cm}$, uma Apuleia sp (LeguminosaeCaesalpinoidae), três Zamia ulei (Zamiaceae), uma Anacardium sp (Anacardiaceae) e um indivíduo não identificado.

A área basal (AB) total encontrada por hectare foi de 22,94 $\mathrm{m}^{2}$ para a área A e $30,27 \mathrm{~m}^{2}$ para a área B. A espécie com a maior área basal foi Inga lateriflora com $5,34 \mathrm{~m}^{2} / \mathrm{ha}$, seguida por Lecythis retusa com 5,22 $\mathrm{m}^{2} /$ ha e Cecropia sp com 5,09 $\mathrm{m}^{2} /$ ha. Os indivíduos não identificados no total das duas parcelas amostradas tiveram $4,15 \mathrm{~m}^{2} / \mathrm{ha}$ de área basal e os indivíduos mortos $2,21 \mathrm{~m}^{2} / \mathrm{ha}$. Inga lateriflora foi a espécie com a maior área basal ocorrida na área $\mathrm{A}$, onde listou-se as 


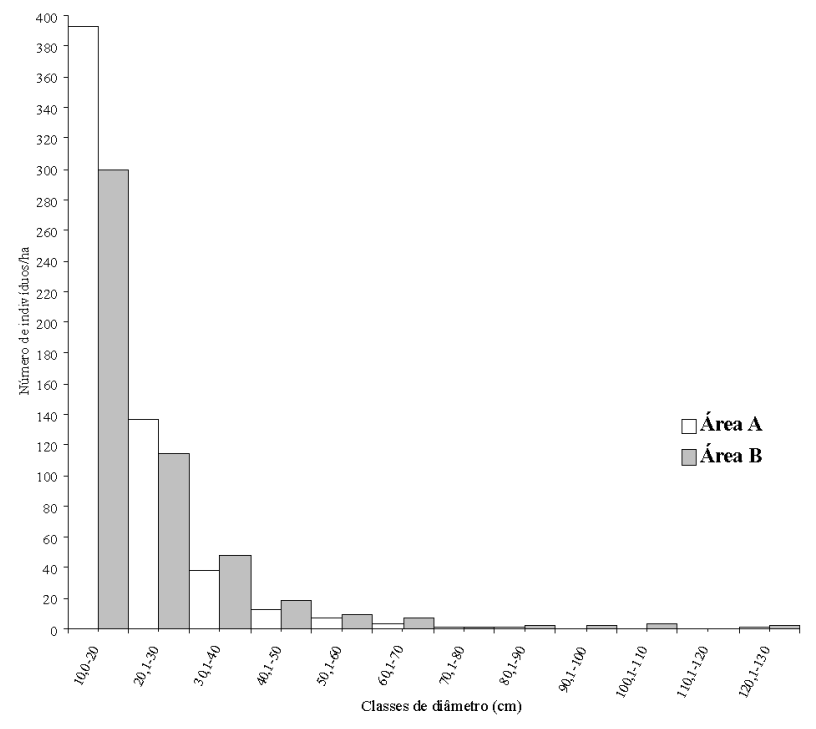

Figura 2 - Distribuição do número de indivíduos, por classes de diâmetro, nos dois hectares amostrados

principais espécies e suas respectivas famílias com maior área basal ocorridas nesta área.

A espécie com o maior volume encontrado foi Zamia ulei com 70,24 $\mathrm{m}^{3}$. Os indivíduos não identificados tiveram um valor para volume de $29,94 \mathrm{~m}^{3}$ e os indivíduos mortos em pé com $15,53 \mathrm{~m}^{3}$.

$\mathrm{Na}$ Tabela 1 estão listadas todas as espécies com o número de indivíduos que ocorreram em cada área. $\mathrm{Na}$ distribuição das espécies, em relação ao número de indivíduos, o levantamento nas duas áreas (A e B) apresentou diversas espécies com mais de 10 indivíduos por hectare e uma grande quantidade de espécies com apenas um indivíduo por hectare.
As famílias que tiveram a maior riqueza em espécies nas duas áreas amostradas estão representadas na Figura 3.

As 20 principais espécies com os maiores índices de valor de importância (IVI) encontradas nas duas áreas estudadas estão respectivamente listados na Tabela 2 e 3, com o número de indivíduos, os valores para densidade, freqüência e dominância relativas, e o índice do valor de cobertura (IVC).

Para a análise de Cluster utilizando o índice de similaridade qualitativo de Sorensen, foram observadas algumas afinidades para a composição de famílias e uma maior similaridade para a composição de gêneros (Fig. 5).

Para a composição de famílias formam grupos os levantamentos: 1) Manaus, Jaru e Caxiuanã e 2) Jaru e Caxiuanã. Os levantamentos de Carajás 1 e Carajás 2 apesar de serem próximos não apresentaram similaridade florística entre as parcelas. As áreas representadas por Ji-Paraná, Tembé e Ka'apor não formaram grupos com nenhum outro levantamento deste trabalho. A composição florística de Presidente Médici e Alta Floresta foram as que mais se diferenciaram não apresentando nenhuma afinidade quanto à composição de famílias com as demais localidades analisadas (Figura 4).

A análise de Cluster realizada com a matriz de presença e ausência de gêneros apresenta uma maior tendência para formação de grupos. Como os três grupos dos levantamentos de: 1) Manaus, Caxiuanã e Jaru, 2) Carajás 1 e Carajás 2 e 3) Tembé e Ka'apor. Os levantamentos de Ji-Paraná, Presidente Médici e Alta Floresta não demonstraram afinidade com outros levantamentos amostrados neste trabalho. Conforme observado, os grupos mais bem definidos são os das parcelas na mesma localidade, como apresentam os dados dos

Tabela 1 - Densidade absoluta das espécies no levantamento dos dois hectares.

\begin{tabular}{|c|c|c|c|c|}
\hline Família & Espécie & Área A & Área B & Total \\
\hline Amaranthaceae & Alternanthera sp & 4 & 0 & 4 \\
\hline \multirow[t]{3}{*}{ Anacardiaceae } & Anacardium sp & 0 & 3 & 3 \\
\hline & Astronium lecointei Ducke & 23 & 0 & 23 \\
\hline & Spondias sp & 1 & 1 & 2 \\
\hline Annonaceae & Annona Montana Macf. Var. Marc. (Mart.) Fr. & 0 & 4 & 4 \\
\hline \multirow[t]{3}{*}{ Apocynaceae } & Aspidosperma polyneuron M. Arg. & 1 & 0 & 1 \\
\hline & Aspidosperma sp 1 & 0 & 1 & 1 \\
\hline & Aspidosperma sp 2 & 0 & 1 & 1 \\
\hline Araliaceae & Didymopanax sp & 0 & 4 & 4 \\
\hline \multirow[t]{4}{*}{ Arecaceae } & Astrocaryum aculeatum G. F. W. Mey. & 14 & 14 & 28 \\
\hline & Attalea maripa (Aubl.) Mart. & 1 & 2 & 3 \\
\hline & Euterpe precatoria Mart. & 4 & 17 & 21 \\
\hline & Socratea exorrhiza (Mart.) H. Wendl. & 3 & 3 & 6 \\
\hline \multirow[t]{2}{*}{ Bignoniaceae } & Jacaranda copaia (Aubl.) D. Don. & 1 & 2 & 3 \\
\hline & Tabebuia serratifolia (Vahl) Nichols & 1 & 2 & 3 \\
\hline
\end{tabular}


Tabela 1 - Continuação

\begin{tabular}{|c|c|c|c|c|}
\hline Família & Espécie & Área A & Área B & Total \\
\hline Bombacaceae & Ceiba sp & 1 & 0 & 1 \\
\hline Boraginaceae & Cordia sp & 5 & 0 & 5 \\
\hline \multirow[t]{3}{*}{ Burseraceae } & Tetragastris altissima (Aubl.) Swart. & 13 & 38 & 51 \\
\hline & Protium sp & 1 & 0 & 1 \\
\hline & Protium sagotianum March. & 1 & 1 & 2 \\
\hline Cecropiaceae & Cecropia sp & 80 & 32 & 112 \\
\hline \multirow[t]{2}{*}{ Euphorbiaceae } & Hevea brasiliensis Muell. Arg. & 1 & 0 & 1 \\
\hline & Pogonophora schomburgkiana Miers ex Benth. & 2 & 0 & 2 \\
\hline \multirow[t]{4}{*}{ Lauraceae } & Ocotea sp 1 & 11 & 7 & 18 \\
\hline & Ocotea sp 2 & 0 & 1 & 1 \\
\hline & Ocotea Iongifolia H.B.K. & 0 & 3 & 3 \\
\hline & Mezilaurus itauba (Meissn.) Taub. Ex Mez. & 2 & 8 & 10 \\
\hline Lecythidaceae & Lecythis retusa Spruce Ex Berg. & 37 & 27 & 64 \\
\hline \multirow[t]{10}{*}{ Leg.-Caesalpinoideae } & Apuleia sp1 & 6 & 0 & 6 \\
\hline & Apuleia sp 1 & 6 & 5 & 11 \\
\hline & Apuleia sp 2 & 2 & 3 & 5 \\
\hline & Apuleia sp 3 & 1 & 0 & 1 \\
\hline & Apuleia sp 4 & 1 & 2 & 3 \\
\hline & Apuleia sp 5 & 2 & 5 & 7 \\
\hline & Bauhinia ungulata L. & 0 & 15 & 15 \\
\hline & Copaifera multijuga Hayne & 0 & 1 & 1 \\
\hline & Dialium guianensis (Aubl.) Sandw. & 2 & 3 & 5 \\
\hline & Hymenaea courbaril L. & 0 & 4 & 4 \\
\hline \multirow[t]{4}{*}{ Leg.-Mimosoideae } & Abarema sp & 0 & 1 & 1 \\
\hline & Albizia subdimiata (Splitg.) Barn.\& Grimes & 2 & 1 & 3 \\
\hline & Inga lateriflora Miquel & 36 & 24 & 60 \\
\hline & Pithecellobium sp & 3 & 8 & 11 \\
\hline \multirow[t]{5}{*}{ Leg.-Papilonoideae } & Andira fraxinifolia Benth. & 37 & 8 & 45 \\
\hline & Dalbergia sp & 1 & 0 & 1 \\
\hline & Hymenolobium pulcherrium Ducke & 1 & 0 & 1 \\
\hline & Swartzia corrugata Benth. & 0 & 1 & 1 \\
\hline & Leguminosae-Papilonoideae sp 1 & 3 & 0 & 3 \\
\hline Malpighiaceae & Byrsonima chrysophylla Marth. & 1 & 0 & 1 \\
\hline Melastomataceae & Bellucia cf. grossularioides (L.) Tr. & 13 & 4 & 17 \\
\hline \multirow[t]{2}{*}{ Moraceae } & Ficus cf. maxima Mill. & 1 & 1 & 2 \\
\hline & Helicostylis podogyne Ducke & 24 & 89 & 113 \\
\hline Myrtaceae & Myrciaria floribunda (Wild.) 0. Berg & 1 & 1 & 2 \\
\hline Polygonaceae & Triplaris brasiliana Cham. & 1 & 0 & 1 \\
\hline Rubiaceae & Kutchubaea sp & 9 & 5 & 14 \\
\hline \multirow[t]{3}{*}{ Rutaceae } & Metrodorea flavida Krause & 5 & 4 & 9 \\
\hline & Zanthoxylum sp 1 & 3 & 15 & 18 \\
\hline & Zanthoxylum sp 2 & 3 & 0 & 3 \\
\hline \multirow[t]{3}{*}{ Sapindaceae } & Pseudima cf. frutiscens (Aubl.) Radlk & 1 & 5 & 6 \\
\hline & Pseudima sp 1 & 0 & 4 & 4 \\
\hline & Pseudima sp 2 & 6 & 1 & 7 \\
\hline \multirow[t]{2}{*}{ Simaroubaceae } & Simarouba amara Aubl. & 2 & 5 & 7 \\
\hline & Simarouba sp & 9 & 10 & 19 \\
\hline Sterculiaceae & Theobroma sylvestris Aubl. Ex. Mart. & 21 & 7 & 28 \\
\hline Strelitziaceae & Phenakospermum guyanense (L.C.Rich.) Endl. & 1 & 0 & 1 \\
\hline
\end{tabular}


Tabela 1 - Continuação

\begin{tabular}{|c|c|c|c|c|}
\hline Família & Espécie & Área A & Área B & Total \\
\hline Tiliaceae & Apeiba tibourbou Aubl. & 1 & 1 & 2 \\
\hline \multirow[t]{2}{*}{ Violaceae } & Amphirrhox sp & 1 & 7 & 8 \\
\hline & Rinoreocarpus ulei (Melch.) Ducke & 43 & 39 & 82 \\
\hline Vochysiaceae & Vochysia sp & 2 & 0 & 2 \\
\hline Zamiaceae & Zamia ulei U. Dammer & 3 & 15 & 18 \\
\hline Não Identificadas & Não Identificadas & 97 & 40 & 137 \\
\hline \multirow[t]{2}{*}{ Mortas em pé } & Mortas em pé & 42 & 0 & 42 \\
\hline & Total & 594 & 507 & 1101 \\
\hline
\end{tabular}

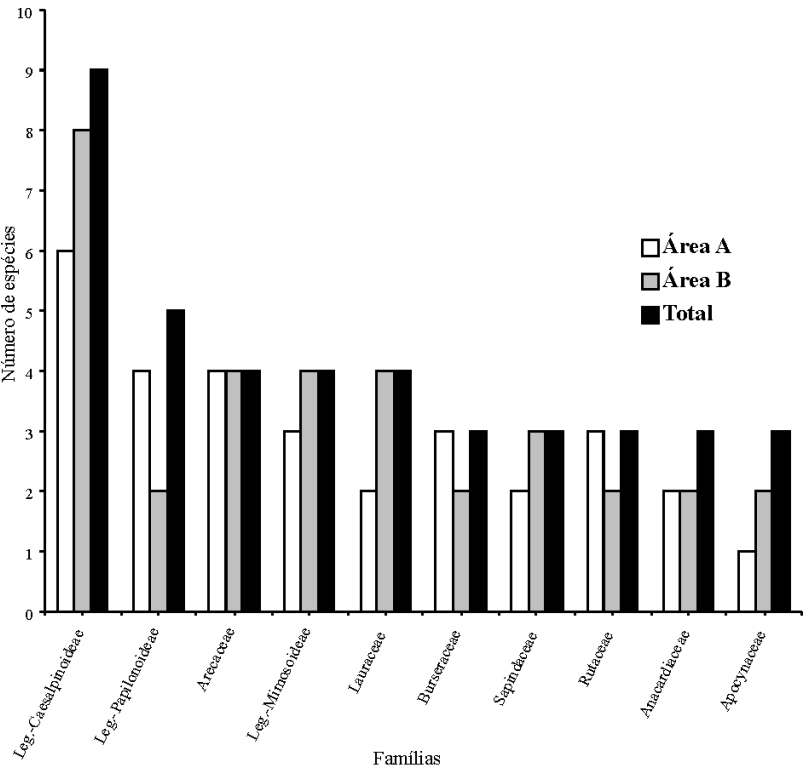

Figura 3 - Riqueza de espécies por famílias, nos dois hectares amostrados.
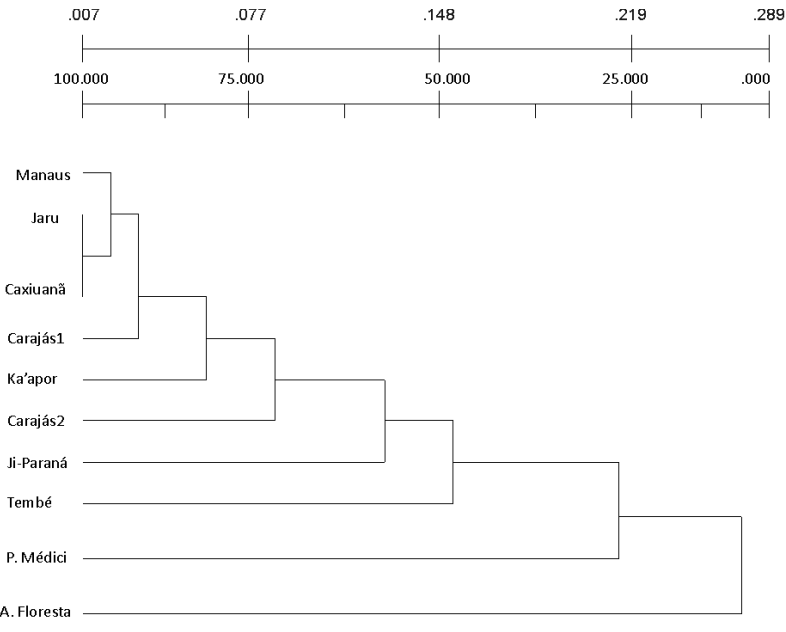

Figura 4 - Agrupamento dos 10 locais da região Amazônica analisadas, pelo método de ligação de média de grupo (UPGMA), aplicados a similaridade de Sorensen, utilizando a matriz de presença e ausência de famílias

Tabela 2 - Lista em ordem decrescente das 20 espécies com maior valor de IVI na área A.

\begin{tabular}{|c|c|c|c|c|c|c|}
\hline Espécie & $\begin{array}{l}\text { Número de } \\
\text { Indivíduos }\end{array}$ & $\begin{array}{l}\text { Densidade } \\
\text { Relativa \% }\end{array}$ & $\begin{array}{l}\text { Freqüência } \\
\text { Relativa \% }\end{array}$ & $\begin{array}{c}\text { Dominância } \\
\text { Relativa \% }\end{array}$ & IVC & IVI \\
\hline Cecropia sp & 80 & 13,47 & 1,75 & 17,28 & 30,74 & 32,50 \\
\hline Não Identificadas & 97 & 16,33 & 1,75 & 14,07 & 30,40 & 32,15 \\
\hline Inga lateriflora & 36 & 6,06 & 1,75 & 17,98 & 24,05 & 25,80 \\
\hline Mortas em pé & 42 & 7,07 & 1,75 & 9,64 & 16,71 & 18,46 \\
\hline Rinoreacarpus ulei & 43 & 7,24 & 1,75 & 5,35 & 12,59 & 14,35 \\
\hline Andira fraxinifolia & 37 & 6,23 & 1,75 & 5,28 & 11,51 & 13,26 \\
\hline Lecythis retusa & 37 & 6,23 & 1,75 & 3,78 & 10,00 & 11,76 \\
\hline Astronium lecointei & 23 & 3,87 & 1,75 & 2,74 & 6,61 & 8,36 \\
\hline Helicostylis podogyne & 24 & 4,04 & 1,75 & 1,89 & 5,93 & 7,68 \\
\hline Astrocarym aculeatum & 14 & 2,36 & 1,75 & 2,77 & 5,13 & 6,88 \\
\hline Theobroma sylvestris & 21 & 3,54 & 1,75 & 1,21 & 4,74 & 6,50 \\
\hline Bellucia grossularioides & 13 & 2,19 & 1,75 & 1,28 & 3,46 & 5,22 \\
\hline Apuleia sp 5 & 2 & 0,34 & 1,75 & 2,74 & 3,08 & 4,84 \\
\hline Tetragastris altissima & 13 & 2,19 & 1,75 & 0,85 & 3,04 & 4,80 \\
\hline
\end{tabular}


Tabela 2 - Continuação

\begin{tabular}{lcccccc}
\hline Espécie & $\begin{array}{c}\text { Número de } \\
\text { Indivíduos }\end{array}$ & $\begin{array}{c}\text { Densidade } \\
\text { Relativa \% }\end{array}$ & $\begin{array}{c}\text { Freqüência } \\
\text { Relativa \% }\end{array}$ & $\begin{array}{c}\text { Dominância } \\
\text { Relativa \% }\end{array}$ & IVC & IVI \\
\hline Ocotea sp 1 & 11 & 1,85 & 1,75 & 1,10 & 2,95 & 4,70 \\
Simarouba sp & 9 & 1,52 & 1,75 & 0,92 & 2,44 & 4,19 \\
Kutchubaea sp & 9 & 1,52 & 1,75 & 0,85 & 2,36 & 4,12 \\
\hline Pseudima sp 2 & 6 & 1,01 & 1,75 & 0,60 & 1,61 & 3,37 \\
Zanthoxylum sp 2 & 3 & 0,51 & 1,75 & 0,92 & 1,43 & 3,18 \\
Apuleia sp 1 & 6 & 1,01 & 1,75 & 0,39 & 1,40 & 3,16 \\
\hline
\end{tabular}

Tabela 3 - Lista em ordem decrescente das 20 espécies com maior valor de IVI na área B.

\begin{tabular}{|c|c|c|c|c|c|c|}
\hline Espécie & $\begin{array}{l}\text { Número de } \\
\text { Indivíduos }\end{array}$ & $\begin{array}{l}\text { Densidade } \\
\text { Relativa \% }\end{array}$ & $\begin{array}{l}\text { Freqüência } \\
\text { Relativa \% }\end{array}$ & $\begin{array}{l}\text { Dominância } \\
\text { Relativa \% }\end{array}$ & IVC & IVI \\
\hline Helicostylis podogyne & 89 & 17,55 & 1,89 & 13,26 & 30,82 & 32,70 \\
\hline Lecythis retusa & 27 & 5,33 & 1,89 & 14,39 & 19,71 & 21,60 \\
\hline Zamia ulei & 15 & 2,96 & 1,89 & 15,28 & 18,24 & 20,13 \\
\hline Rinoreocarpus ulei & 39 & 7,69 & 1,89 & 4,98 & 12,67 & 14,55 \\
\hline Não Identificadas & 40 & 7,89 & 1,89 & 3,05 & 10,93 & 12,82 \\
\hline Cecropia sp & 32 & 6,31 & 1,89 & 3,74 & 10,05 & 11,93 \\
\hline Tetragastris altissima & 38 & 7,50 & 1,89 & 1,99 & 9,49 & 11,37 \\
\hline Inga lateriflora & 24 & 4,73 & 1,89 & 4,04 & 8,77 & 10,66 \\
\hline Anacardium sp & 3 & 0,59 & 1,89 & 4,57 & 5,16 & 7,05 \\
\hline Apuleia sp 4 & 2 & 0,39 & 1,89 & 4,58 & 4,98 & 6,87 \\
\hline Astrocaryum aculeatum & 14 & 2,76 & 1,89 & 1,81 & 4,57 & 6,46 \\
\hline Pithecellobium sp & 8 & 1,58 & 1,89 & 2,50 & 4,08 & 5,97 \\
\hline Euterpe precatoria & 17 & 3,35 & 1,89 & 0,73 & 4,08 & 5,97 \\
\hline Bauhinia ungulata & 15 & 2,96 & 1,89 & 0,94 & 3,90 & 5,78 \\
\hline Zanthoxylum sp 1 & 15 & 2,96 & 1,89 & 0,93 & 3,89 & 5,78 \\
\hline Simarouba sp & 10 & 1,97 & 1,89 & 1,00 & 2,97 & 4,86 \\
\hline Mezilaurus itauba & 8 & 1,58 & 1,89 & 1,33 & 2,91 & 4,80 \\
\hline Hymenaea courbaril & 4 & 0,79 & 1,89 & 1,99 & 2,78 & 4,67 \\
\hline Pseudima sp 1 & 4 & 0,79 & 1,89 & 1,66 & 2,45 & 4,34 \\
\hline Andira fraxinifolia & 8 & 1,58 & 1,89 & 0,81 & 2,39 & 4,27 \\
\hline
\end{tabular}

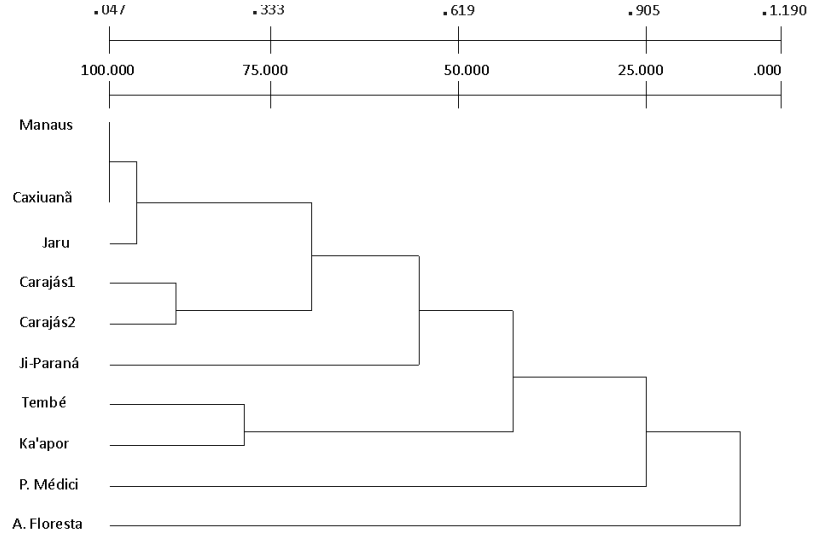

Figura 5 - Agrupamento dos 10 locais da região Amazônica analisadas, pelo método de ligação de média de grupo (UPGMA), aplicados a similaridade de Sorensen, utilizando a matriz de presença e ausência de gêneros levantamentos da composição florística para gêneros de Carajás 1 e Carajás 2 (Figura 5).

O resultado da análise multivariada de NMS (Escalonamento multidimensional não-métrico), para ordenação dos grupos por meio do índice qualitativo de Jaccard, utilizando as matrizes de presença e ausência de famílias (Figura 6), demonstrou que os levantamentos de Alta Floresta e Presidente Médici são os mais distantes. Forma-se grupo os levantamentos de Ka'apor e Carajás 2 juntamente com o de Tembé, apesar de estar um pouco distante. $\mathrm{O}$ outro grupo com maior afinidade entre as composições de famílias foi do levantamento de Manaus, Caxiuanã, Jaru, Carajás 1 e Ji-Paraná, observando uma relação mais acentuada entre as florestas de Manaus, Caxiuanã e Jaru, apesar de estarem distantes geograficamente.

A análise multivariada NMS aplicada para a presença e ausência de gêneros utilizando o índice qualitativo de Jaccard, 
mostra também um distanciamento dos levantamentos de Alta Floresta e Presidente Médici. Os levantamentos de Carajás2, Manaus, Caxiuanã e Jaru formam um grupo. Os levantamentos de Carajás1, Ji-Paraná, Tembé e Ka’apor formam outro grupo (Figura 7).

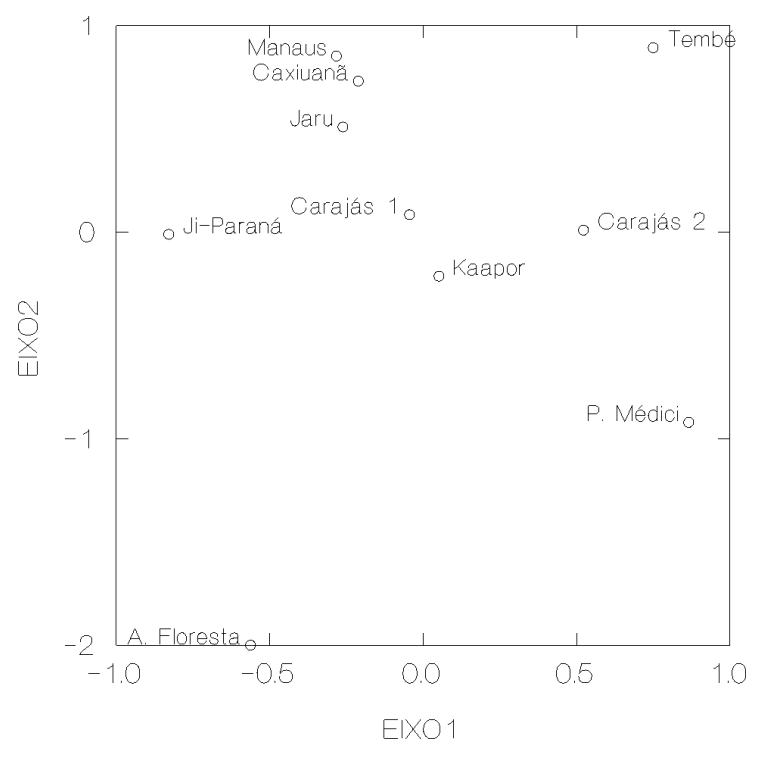

Figura 6 - Análise de NMS das localidades aplicada a presença e ausência de famílias para os 10 levantamentos analisados.

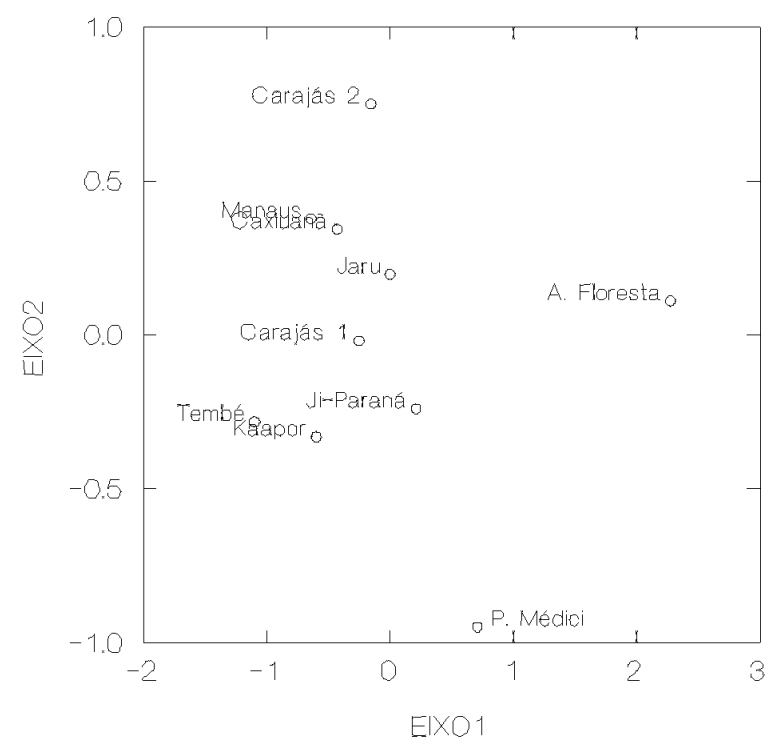

Figura 7 - Análise de NMS das localidades aplicada a presença e ausência de gêneros para os 10 levantamentos analisados.

\section{DISCUSSÃO}

Os 1101 indivíduos amostrados pertencentes a 32 famílias, 54 gêneros e 68 espécies nos dois hectares deste levantamento estão abaixo da média para a Amazônia. Isso pode ocorrer pelo fato de que 137 indivíduos encontrados não foram identificados, ocasionado influência no número de famílias, gêneros e espécies deste trabalho.

Apenas considerando os parâmetros quanto ao número de indivíduos e famílias encontrados neste trabalho, estes se assemelham a média encontrada na Amazônia, como em Carajás-PA por Salomão et al. (1988), apresentando 484 indivíduos e 39 famílias, em Tembé-PA por Balée (1987) onde obteve uma amostra de 456 indivíduos e 35 famílias, em Ka'apor-MA Balée (1986) que levantou 498 indivíduos e 35 famílias, e em Presidente Médici-RO, Maciel \& Lisboa (1989) amostraram 602 indivíduos e 33 famílias.

Com exceção dos 137 indivíduos não identificados nos dois hectares do presente levantamento, que obtiveram o mais alto valor de IVI nas duas áreas de coleta, observa que os dados para o índice de valor de importância (IVI) são parecidos com resultados citados, destacam-se as famílias Cecropiaceae, Moraceae e Leguminosae-Mimosoideae como as que tiveram valores expressivos para o índice.

O resultado da análise multivariada de NMS (Escalonamento multidimensional não-métrico), para ordenação dos grupos por meio do índice qualitativo de Jaccard, utilizando as matrizes de presença e ausência de famílias apresentou uma formação de grupos por regiōes. No caso de Alta Floresta pode-se apontar que fatores como o meio possa estar influenciando na composição florística, o que pode determinar a existência de uma diversidade um tanto quanto diferente das outras regiōes aqui estudadas, destacando similaridade apenas com o estudo realizado em Presidente Médici-RO.

Oliveira (1997) contatou que a utilização de diversos trabalhos na Amazônia demonstra que existe um elevado percentual de espécies vegetais com baixa densidade, dificultando as inúmeras metodologias utilizadas para as comparações entre os diferentes estudos, pois pouco se conhece sobre as diferenças regionais e em larga escala entre os padrões estruturais.

A área estudada no município de Alta Floresta possui alguns fatores que podem explicar o não agrupamento com outras regiões que foram analisadas neste trabalho, evidenciando o fato de ter um elevado número de indivíduos não identificados, sendo que estes poderiam contribuir na riqueza e diversidade de espécies.

Um dos fatores é a presença um tanto quanto significativa de árvores características de áreas degradadas, como as do gênero Cecropia sp. e da espécie Helicostylis podogyne Ducke 
na região amostrada, o mesmo que aconteceu no levantamento de Presidente Médici-RO, que segundo Maciel \& Lisboa (1989), houve a predominância da espécie Guadua superba Hub. (Graminae).

Oliveira (1997) discute que a distinção entre as florestas se dá, principalmente, por ocorrer uma maior dominância na comunidade por determinadas espécies, o que normalmente não apresenta contribuição nas florestas maduras, mas também por uma baixa semelhança florística ao nível genérico.

\section{CONCLUSÕES}

As áreas amostradas no trabalho realizado no município de Alta Floresta-MT apresentaram um baixo número de indivíduo em sua composição florística inferior à média para uma floresta ombrófila aberta tropical.

A presença da família Cecropiaceae com alto valor do IVI indica a influência na composição e estrutura florística local, uma vez que os dois fragmentos estudados estão localizados na área de uma fazenda com pastagens para gado.

Não houve agrupamento, quanto à riqueza e diversidade de espécies, com outras regióes que analisadas neste trabalho.

Somente os parâmetros quanto ao número de indivíduos e famílias apresentaram uma similaridade com as áreas levantadas em Carajás-PA, Tembé-PA, Ka’apor-MA e em Presidente Médici-RO.

\section{AGRADECIMENTOS}

Ao convênio interinstitucional INPA/CAPES/UNEMAT por ter me proporcionado à oportunidade, de fazer parte do curso de Mestrado em Ecologia do Programa de Integrado de Pós-graduação em Biologia Tropical e Recursos Naturais no INPA. A Dra. Tânia Sanaiotti pela colaboração e incentivo.

\section{BIBLIOGRAFIA CITADA}

Balée, W. 1986. Análise Preliminar de Inventário Florestal e a Etnobotânica Ka’apor (Maranhão). BoL Mus. Para. Enúlio Goeldi, Sér. Boi., 2(2): 141-167.

Balée, W. 1987. A Etnobotânica Quantitativa dos Índios Tembé (Rio Ourupi, Pará). Bol Mus. Para. Enúlio Goeldi, Sér. BoL, 2(1): 29-50.

Belbin, L. 1995. PATN-Pattern Analysis Package: Technical Reference. Division of Wildlife and Ecology .CSIRO. Australia.

Cesar, O.; Leitão-Filho, H. de F. 1990. Estudo Fitossociológico de Mata Mesófila Semidecídua na Fazenda Barreiro Rico, Município de Anhembi, SP. Revista Bras. Biol, 50 (2): 443-452.

Cunha, N.G.; Oliveira, V.A.; Oenning, I.; Souza, L.F.P. 1980. Pedologia. In: RADAMBRASIL -Levantamento de Recursos Naturais, Folha Sc. 21 Juruena -Rio de Janeiro MME. 165-324 p.
Fortin, M.I.; Gurevitch, I. 1983. Mantel Tests: Spatial Structure in Field Experiments. In: Scheiner, S.M; Gurevitch, I. (Eds.). Design and Analysis o/ Ecological Experiments. New York, USA. 342-359p.

Gentry .A.H.; Emmons, L.H. 1987. Geographical Variation in Fertility, Phenology, and Composition of the Understory of Neotropical Forests. Biotropica, 19(3): 216-227.

Hubbell, S.P. 1979. Tree Dispersion, Abundance and Diversity in a Tropical Dry Forest. Science. 203(4387): 129-1309.

Hubbell, S.P.; Foster, R.B. 1986. Commonness and Rarity in a Neotropical Forest: Implications for Tropical Tree Conservation. In: Soulé, M. E. (Ed.). Conservation Biology: The Science of Scarcity and Diversity. Massachusetts, Sinauer Assoc. Inc, Publ. $584 \mathrm{p}$.

IBGE - Instituto Brasileiro de Geografia e Estatística 2006. Disponível em www.ibge.gov.br/home/geociencias/cartogramas/relevo. html. Acesso em 13 out. 2008.

INPA/CPPF. 1991. Catálogo de Madeiras da Amazônia. Características Tecnológicas; Área da Hidrelétrica de Balbina. Manaus. 163 pp.

Jardim, F.C.S.; Hosokawa, R.T. 1986. Estrutura da Floresta Equatorial Úmida da Estação Experimental de Silvicultura Tropical do INPA. Acta Amazonica, 16/17 (único): 411-508.

Joly, A.B. 1991. Botânica: Introdução à Taxonomia VegetaL Editora Nacional (Biblioteca Universitária. Série 3. Ciências puras; v. 4). São Paulo. $777 \mathrm{pp}$.

Lorenzi, H. 1992. Arvores Brasileiras: Manual de Identificação e Cultivo de Plantas Arbóreas Nativas do BrasiL Vol. 02. Editora Plantarum. Nova Odessa, São Paulo. 352 p.

Loureiro, R.L.; Dias, A.A.; Magnago, H. 1980. Vegetação. In: RADAMBRASIL - Levantamento de Recursos Naturais, Folha Sc. 21 Juruena -Rio de Janeiro MME. 325-376p.

Mabberley, D.J. 1981. The Plant-Book. Cambridge University Press. New York, USA.

Maciel, U.N.; Lisboa, P.L.B. 1989. Estudo Florístico de 1 Hectare de Mata de Terra Firme no Km 15 da Rodovia Presidente Médici -Costa Marques (RO-429), Rondônia. BoL Mus. Para. Enúlio Goeldi, Sér. BoI., 5(2): 25-37.

McCune, H. ; Mefford, M.J. 1995. PC-ORD. Multivariate Analysis o/ Ecological Data, Version 2.0. MjM Software Design, Gleneden Heach, Oregon, USA. 126pp.

Oliveira, A.A. 1997. Diversidade, Estrutura e Dinâmica do Componente Arbóreo de uma Floresta de Terra Firme de Manaus, Amazonas. Tese de Doutorado, Universidade de São Paulo. São Paulo, São Paulo. 187pp.

Parrotta, I.A.; Francis, I.K.; AJmeida, R.R. 1995. Trees os the Tapajósa photografic field guide. Gen. Tech. Rep. IITF-1. Rio Píedras, PR: U.S. Department of Agricuhure, Forest Service, International Institute of Tropical Forestry. 370pp.

Pott, A.; Pott, V.I. 1994. Plantas do Pantanal Empresa Brasileira de Pesquisas Agropecuária, Centro de Pesquisa Agropecuária do Pantanal. Corumbá, MS. EMBRAP A- SPI. 320pp. 
Prefeitura Municipal de Alta Floresta. 1999. Secretaria Municipal de Agricultura. Dados Pluviométricos de Alta Floresta. Alta Floresta, MT.

Ribeiro, R.I.; Higuchi, N.; Azevedo, C.P. 1993. Estudos Fitossociológicos nas Regiōes de Carajás e Marabá-PA Monografia, UTAM, Manaus, Amazonas. 90pp.

Ribeiro, I.E.L.S.; Hopkins, M.I.G.; Vicentini, A.; Sothers, C.A.; Costa, M.A.S.; Brito, I. M.; Souza, M.A.D.; Martins, L.H.P.; Lohmann, L.G.; Assunção, P.A.C.L.; Pereira, E. C.; Silva, C.F.; Mesquita, M.R.; Procópio, L.C. 1999a. Flora da Reserva Ducke: Guia de Identificação das Plantas Vasculares de uma Floresta de Terra-Firme na Amazônia Central. Instituto Nacional de Pesquisas da Amazônia (INPA). Manaus, Amazonas. 816p. il.

Rizzini, C.T. 1971. Árvores e Madeiras Úteis do Brasil: Manual de Dendrologia Brasileira. Ed. Edgard Blucher. São Paulo, SP. $294 \mathrm{pp}$.

Rossi L.M.B. ; Higuchi, N. 1998. Comparação Entre Métodos de Análise do Padrão Espacial de Espécies Arbóreas de Uma Floresta Tropical Úmida. In: Gascon, C\& Moutinho, P. (Eds.). Floresta Amazônica: Dinâmica, Regeneração e Manejo. Instituto Nacional de Pesquisas da Amazônia (INPA). Manaus, Amazonas. p.41-59.

RuokoIainen, K.; Tuomisto, H. 1998. Vegetación Natural de la Zona de Iquitos. In: Kalliola, R.; Flores, P.S. (Eds.). Geoecologia y Desarrollo Amazônico: Estudio Integrado em Ia Zona de Iquitos. Annales Universitatis Turkuensis. Ser. A II. Peru. 114: 253365.
Sánchez, R.O. 1992. Zoneamento Agroecológico do Estado de Mato Grosso: Ordenamento Ecológico-Paisagístico do Meio Natural e Rural. Cuiabá, Mato Grosso, Fundação de Pesquisas Cândido Rondon. 160p. il.

Santos, I. 1996. Análise de Modelos de Regressão para Estimar a Fitomassa da Floresta Tropical Úmida de Terra-Firme da Amazônia Brasileira. Tese de Doutorado, Universidade Federal de Viçosa. Viçosa, Minas Gerais. 121pp.

Shepherd, G.I. 1994. FITOPAC 1. Manual de Usuários. Departamento de Botânica, UNICAMP. Campinas, São Paulo.

Terborgh, I.; Andresen, E. 1998. The Composition of Amazonian Forests: Patterns at Local and Regional Scales. Journal of Tropical Ecology, 14: 645-664.

Valentin, I.L. 1995. Agrupamento e Ordenação. In: Peres-Neto, P.R.; Valentin, I.L. \& Femandes, F.A.S. (Eds). Tópicos em Tratamento de Dados Biológicos. Oecologia Brasiliensis, Rio de Janeiro. 2: 27-55.

Vieira, O.; Hosokawa, R.T. 1989. Composição Florística da Vegetação da Regeneração Natural. 1 Ano após Diferentes Níveis de Exploração de uma Floresta Tropical Úmida. Acta Amazonica. 19(Único): 401-413.

Wilkinson, L. 1990. SYSTAT: The System for Statistics. SYSTATInc. Evanston, Illinois, USA.

Recebido em 05/05/2008

Aceito em 19/10/2008 